\title{
Estudio del endurecimiento superficial por láser de fundiciones grises
}

\author{
A.L. Maco ${ }^{(*)}$ y F.J. Belzunce ${ }^{(*)}$ \\ Resumen Se ha realizado el endurecimiento superficial con láser de tres fundiciones grises de diferente compo- \\ sición química, cuantificándose las variaciones microestructurales introducidas, las profundidades \\ afectadas por el tratamiento y la variación de dureza lograda al modificar tanto la densidad de poten- \\ cia como la velocidad de pasada. Siempre que la densidad de energía aportada con el láser supere un \\ cierto valor crítico, se obtiene una capa superficial que llega a fundir en el tratamiento, dando lugar a \\ una estructura ledeburítica de muy alta dureza, obteniéndose, por debajo de ella, una segunda capa, \\ también muy dura, de fundición martensítica.
}

Palabras clave: Endurecimiento con láser. Fundiciones grises. Tratamiento superficial. Evolución microestructural. Dureza superficial.

\section{Laser surface hardening of gray cast irons}

\begin{abstract}
Three different ferritic-pearlitic gray cast irons have been laser hardened. The influence of laser power density and scanning speed on their microstructural modification, affected depth and surface hardness have been evaluated. It was observed that when the laser energy density surpasses a certain critical value, a surface layer melts and a ledeburitic microstructure of very high hardness is obtained. Below it, a second layer of martensitic microstructure also of high hardness, is produced.
\end{abstract}

\section{Keywords: Laser hardening. Gray cast irons. Surface treatment. Microstructural modification.} Surface hardness.

\section{INTRODUCCIÓN}

La resistencia al desgaste es una de las propiedades más importantes que afectan a la vida de las fundiciones férreas en muchas de sus aplicaciones y la ejecución de tratamientos de endurecimiento superficial es una de las técnicas más eficientes para mejorar el comportamiento de estos productos. Por otro lado, los láseres industriales tienen una aplicación creciente para el procesado metalúrgico y, más concretamente, para el endurecimiento por transformación de la superficie de aceros y fundiciones de hierro ( 1 y 2 ).

Para iniciar cualquier proceso de tratamiento superficial con láser, es preciso controlar adecuadamente varios parámetros del láser modificables por el usuario, como son la potencia aplicada sobre la superficie, el tamaño del haz láser, la velocidad relativa haz-pieza, el diámetro de la boquilla de

(*) Dpto. de Materiales. E.T.S.I. Industriales. Campus Universitario (Univ. de Oviedo). 33203-Gijón (España). salida de los gases de protección, el modo electromagnético de salida del haz, etc., que, junto con las propiedades térmicas del material a tratar, van a permitir calcular la distribución de temperaturas en la pieza o probeta. Como resultado de esto, se pueden predecir los efectos del tratamiento láser en cuanto a la profundidad de la capa susceptible de ser modificada, evitando así tener que recurrir al método experimental de prueba y error. Estas estimaciones se hicieron en este trabajo utilizando el modelo unidimensional de flujo de calor. Por otra parte, se hizo un análisis metalográfico del material después del tratamiento con láser y se cuantificó el endurecimiento introducido y la profundidad afectada mediante medidas de dureza.

\section{PROCEDIMIENTO EXPERIMENTAL}

El trabajo experimental se realizó utilizando tres tipos de fundiciones grises de matriz ferrito-perlítica: fundición gris laminar $(L)$, fundición gris nodu$\operatorname{lar}(N)$ y fundición gris nodular aleada $(N A)$. 
La composición química de estos materiales se muestra en la tabla I. De acuerdo con los valores del carbono equivalente $(C E)$ de cada una de las fundiciones, podemos decir que las fundiciones laminar y nodular aleada son hipoeutécticas, mientras que la fundición nodular es ligeramente hipereutéctica. De cualquier modo, la composición de cada una de estas tres fundiciones está muy próxima a la del punto eutéctico. Igualmente existen diferencias significativas en el contenido de silicio de las tres fundiciones.

A continuación, la tabla II muestra los valores de la densidad $(\rho)$, calor específico $(c)$, conductividad térmica $(K)$ y difusividad térmica $(\alpha)$ de cada uno de los materiales usados (3). Estos valores se utilizarán en la aplicación del modelo de flujo unidireccional de calor con el fin de predecir las modificaciones microestructurales que se generarán en cada uno de estos materiales, ya que sabemos que la absorción y transmisión del calor aportado por el tratamiento láser depende de estas constantes.

Las probetas empleadas en las distintas pruebas y análisis realizados en este estudio han sido obtenidas por mecanizado a partir de bloques de $200 \times$ $350 \times 150 \mathrm{~mm}$, directamente fundidos, sin tratamiento térmico adicional (4).

Todas las probetas fueron recubiertas con una pintura negra mate, para aumentar el poder de absorción de la radiación láser y disminuir, de este modo, en la medida de lo posible, las pérdidas de potencia por reflexión de la energía láser.

Se ha utilizado un láser de $\mathrm{CO}_{2}$ de $1.800 \mathrm{~W}$. Las condiciones de operación del láser fueron las siguientes:

- La presión de argón (gas de protección) utilizada fue de 0,5 bares.

- La potencia teórica a la que se hizo trabajar el equipo fue del $100 \%$.

- La boquilla de salida del haz láser usada fue de $2 \mathrm{~mm}$.

TABLA I.- Composición química (\% en masa)

TABLE I.-Chemical composition (mass \%)

\begin{tabular}{|c|c|c|c|}
\hline$\%$ & $\mathrm{~L}$ & $\mathrm{~N}$ & N.A. \\
\hline $\mathrm{C}$ & 3,420 & 3,500 & 3,290 \\
$\mathrm{Si}$ & 2,010 & 2,960 & 2,420 \\
$\mathrm{Mn}$ & 0,490 & 0,210 & 0,160 \\
$\mathrm{Mo}$ & 0,090 & 0,010 & 0,240 \\
$\mathrm{Cu}$ & 0,017 & 0,110 & 1,070 \\
$\mathrm{~S}$ & 0,088 & 0,008 & 0,007 \\
$\mathrm{P}$ & - & 0,035 & 0,020 \\
$\mathrm{Cr}$ & 0,100 & 0,060 & 0,031 \\
$\mathrm{Al}$ & - & 0,019 & 0,051 \\
$\mathrm{Mg}$ & - & 0,021 & 0,070 \\
$\mathrm{CE}$ & 4,09 & 4,498 & 4,100 \\
\hline
\end{tabular}

TABLA II.- Constantes físicas de los materiales

TABLE II.- Physical constants

\begin{tabular}{|l|c|c|c|c|}
\hline & $\begin{array}{c}\rho \\
\left(\mathrm{g} / \mathrm{cm}^{3}\right)\end{array}$ & $\begin{array}{c}\mathrm{c} \\
(\mathrm{J} / \mathrm{kg} \mathrm{K})\end{array}$ & $\begin{array}{c}\mathrm{K} \\
(\mathrm{W} / \mathrm{m} \mathrm{K})\end{array}$ & $\begin{array}{c}\alpha \\
\left(\mathrm{cm}^{2} / \mathrm{s}\right)\end{array}$ \\
\hline F. LAMINAR & 7,000 & 544,3 & 50,00 & 0,1312 \\
F. NODULAR & 7,120 & 603,0 & 31,15 & 0,0725 \\
F. N. ALEADA & 6,925 & 544,3 & 27,70 & 0,0735 \\
\hline
\end{tabular}

- Se trabajó con tres diámetros del haz láser: 7,10 y $14 \mathrm{~mm}$.

- Se utilizaron velocidades de pasada comprendidas entre 250 a $1.200 \mathrm{~mm} / \mathrm{min}$, incrementándose ésta de 50 en 50 unidades.

Para la observación metalográfica se utilizó un microscopio óptico Nikon y las medidas de microdureza se efectuaron con un penetrador Vickers y carga de $0,5 \mathrm{~kg}$.

\section{RESULTADOS Y DISCUSIÓN}

\subsection{Cálculo teórico de las profundidades transformadas}

Si se estima que el flujo de calor discurre sólo en una dirección (espesor de la probeta) y no hay convección o generación de calor y si se asume, igualmente, que hay un elevado calor superficial aportado y que éste es constante y que, además, las propiedades térmicas también son constantes. Si por otra parte, no hay pérdida de calor por radiación o fusión, entonces se puede calcular la temperatura máxima para una profundidad determinada mediante la siguiente ecuación (5):

$$
T_{z, t}=T_{0}+\frac{2 F_{0}}{K}\left\{\sqrt{\alpha \operatorname{\alpha ierfc}\left(\frac{z}{2 \sqrt{\alpha t}}\right)}\right\}
$$

Si se deja de aplicar potencia, entonces el material se enfriará de acuerdo con la relación:

$$
T_{z, t}=\frac{2 F_{0}}{K} \sqrt{\alpha\left[\sqrt{\operatorname{tierfc}\left(\frac{z}{2 \sqrt{\alpha t}}\right)}-\sqrt{t_{1}-\operatorname{tierfc}\left(\frac{z}{2 \sqrt{\alpha\left(t_{1}-t\right)}}\right)}\right]}
$$

donde: $T$ es la temperatura en ${ }^{\circ} \mathrm{C}, z$ es la profundidad en $\mathrm{cm}, t$, el tiempo de interacción en $\mathrm{s} ; t_{1}$, el tiempo a partir del cual se deja de aplicar potencia, en $\mathrm{s} ; K$, la conductividad térmica en $\mathrm{W} / \mathrm{cm}{ }^{\circ} \mathrm{C} ; \alpha$, difusividad térmica en $\mathrm{cm}^{2} / \mathrm{s} \mathrm{y}_{0}$, la densidad de potencia absorbida en $\mathrm{W} / \mathrm{cm}^{2}$. 
Utilizando las fórmulas del modelo de flujo unidireccional de calor expuestas, se calcularon los espesores transformados que se obtienen al utilizar unas condiciones de láser determinadas, tales como la potencia del láser, el diámetro del haz y las velocidades de pasada.

El cálculo de los espesores transformados o profundidad a la que se supera la temperatura de transformación austenítica, $738{ }^{\circ} \mathrm{C}$, se hizo usando la expresión [1], asumiendo una potencia de trabajo de $1.800 \mathrm{~W}$ y un diámetro del haz láser de $14 \mathrm{~mm}$.

La figura 1 muestra que los espesores transformados que predice el modelo en las tres fundiciones son muy similares. Únicamente se destaca el mayor espesor transformado que predice el modelo para la fundición nodular aleada, lo que es lógico, dado el valor de la conductividad térmica, $K$, que en esta fundición es menor. Fuera de esto, los valores calculados evolucionan dentro de lo esperado, es decir, conforme aumenta la velocidad de pasada el espesor transformado disminuye.

\subsection{Profundidad afectada real}

A pesar de que el modelo predice unos espesores transformados suficientemente amplios para un diámetro del haz láser de $14 \mathrm{~mm}$ en los tres materiales, se han realizado pruebas experimentales aumentando la densidad de potencia, es decir, empleando además del diámetro de $14 \mathrm{~mm}$, diámetros del haz láser de 7 y $10 \mathrm{~mm}$. Para esto, hay una

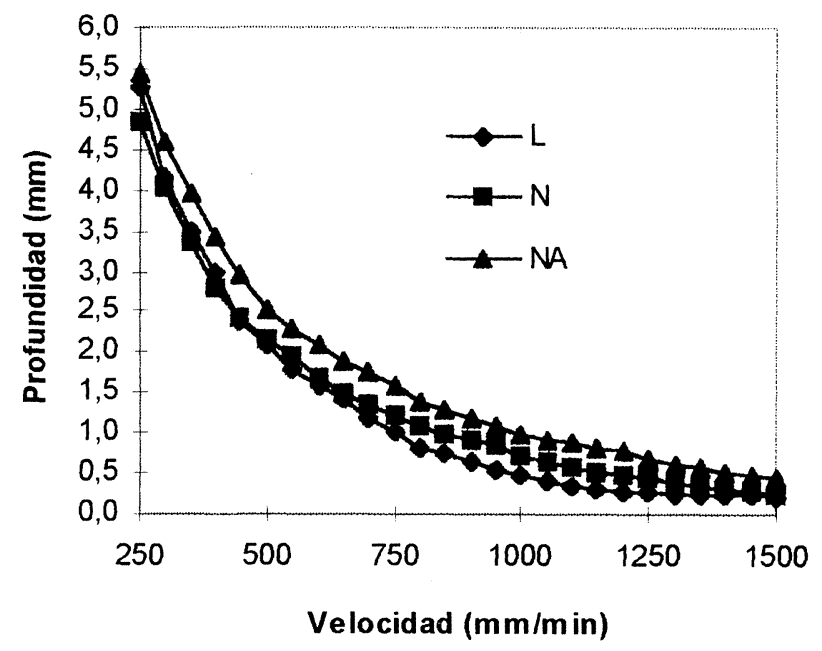

FIG. 1.- Evolución de la profundidad afectada en función de la velocidad para los tres tipos de fundición usando un diámetro del haz láser de $14 \mathrm{~mm}$ y una potencia de $1.800 \mathrm{~W}$.

FIG. 1.- Heat affected depth vs scanning speed for the three types of cast irons (laser beam diameter: $14 \mathrm{~mm}$, power $1.800 \mathrm{~W}$ ). razón muy importante, ya que en todos los cálculos se ha considerado una potencia absorbida de 1.800 $\mathrm{W}$, que es la potencia real suministrada por el equipo cuando se trabaja al $100 \%$, pero la potencia absorbida por el material, aun cuando éste se recubra con una película antirreflectante, será algo inferior y, en consecuencia, los espesores transformados serán menores que los pronosticados por el modelo.

Después del tratamiento superficial por láser aplicado a las probetas en las condiciones estipuladas en el apartado anterior, se procedió al corte transversal de las mismas, a su preparación metalográfica y, posteriormente, a la observación microscópica para determinar los espesores afectados térmicamente. Se distinguieron 2 zonas: una zona fundida (que en el enfriamiento posterior se transforma en fundición blanca, $F B$ ) y una zona templada (transformada en austenita y luego en martensita, ZAT). Se midieron ambos espesores y se determinó la profundidad total alterada por el tratamiento, $z$.

La figura 2 muestra que el espesor alterado térmicamente tiende a ser mayor cuanto menor es el diámetro del haz láser. Sin embargo, la gran diferencia entre usar uno u otro diámetro de haz láser no radica en los espesores transformados, sino más bien en la homogeneidad de la huella. Cuando se emplea el diámetro más grande, de $14 \mathrm{~mm}$, la huella obtenida abarca todo el ancho de la probeta y, además, el espesor transformado es prácticamente paralelo a la superficie tratada.

Se compara, también, la respuesta frente al tratamiento superficial por láser de las tres fundiciones. Cuando se tratan las tres fundiciones con un diámetro de 7, 10 ó $14 \mathrm{~mm}$, los espesores transformados, en el caso de la fundición laminar, son

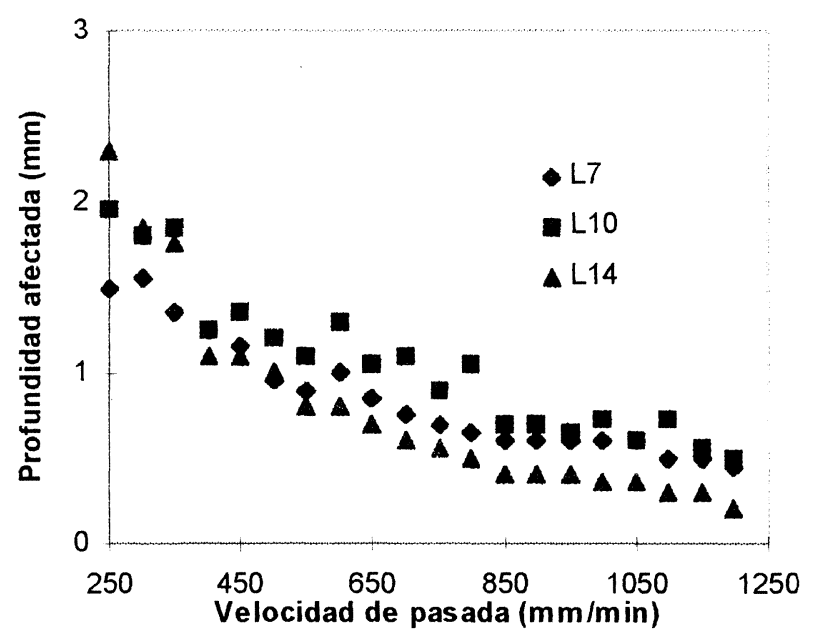

FIG. 2.- Evolución de la profundidad afectada térmicamente en la fundición laminar.

FIG. 2.- Heat affected depth in laminar gray iron. 
sensiblemente menores que los de las otras dos fundiciones, en virtud de su mayor difusividad térmica, mientras que las dos fundiciones nodulares presentan espesores transformados muy similares (Fig. 3).

\subsection{EVOLUCION MICROESTRUCTURAL}

La figura 4 muestra la variación microestructural típica completa en una fundición nodular tratada con láser $(1.800 \mathrm{~W}$ de potencia y una velocidad de pasada de $250 \mathrm{~mm} / \mathrm{min}$ ). En ella, se aprecia claramente cómo evoluciona la estructura a medida que es afectada por el calor aportado por el láser. En la parte superior, se distingue la zona fundida, que todavía conserva nódulos de grafito que no fueron completamente disueltos. Luego aparece una interfase entre la fundición blanca y la siguiente región constituida por martensita y austenita retenida. Si se sigue bajando, se puede ver una capa constituida por nódulos de grafito embebidos en una matriz martensítica, la cual, además, muestra un carácter no homogéneo (zonas oscuras y claras). A continuación, se encuentra otra interfase entre la matriz martensítica y la matriz original y, finalmente, aparece, en la zona inferior de la figura, el material original no afectado por el tratamiento láser.

Esta secuencia de estructuras es muy similar en todos los materiales tratados. Se destaca, sin embargo, que al utilizar un haz láser de 10 ó $14 \mathrm{~mm}$ de diámetro no se llega a fundir la superficie de las probetas (en los tres tipos de fundiciones) cuando la velocidad de pasada supera un cierto límite $(1.100 \mathrm{y}$ $550 \mathrm{~mm} / \mathrm{min}$ para los haces de 10 y $14 \mathrm{~mm}$ de diámetro, respectivamente).

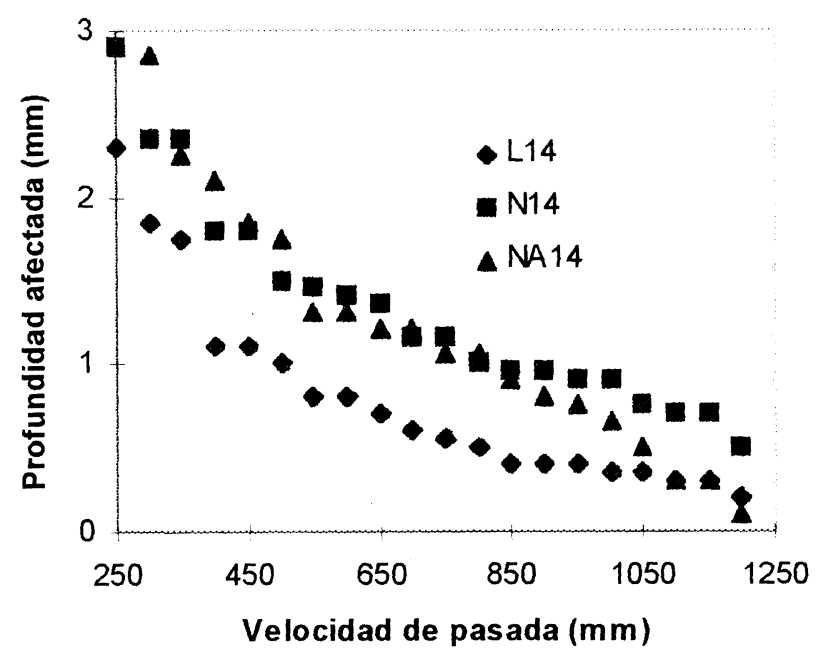

FIG. 3.- Evolución de la profundidad afectada para un rayo del haz láser $14 \mathrm{~mm}$ de diámetro.

FIG. 3.- Heat affected depth. Laser beam diameter: $14 \mathrm{~mm}$.

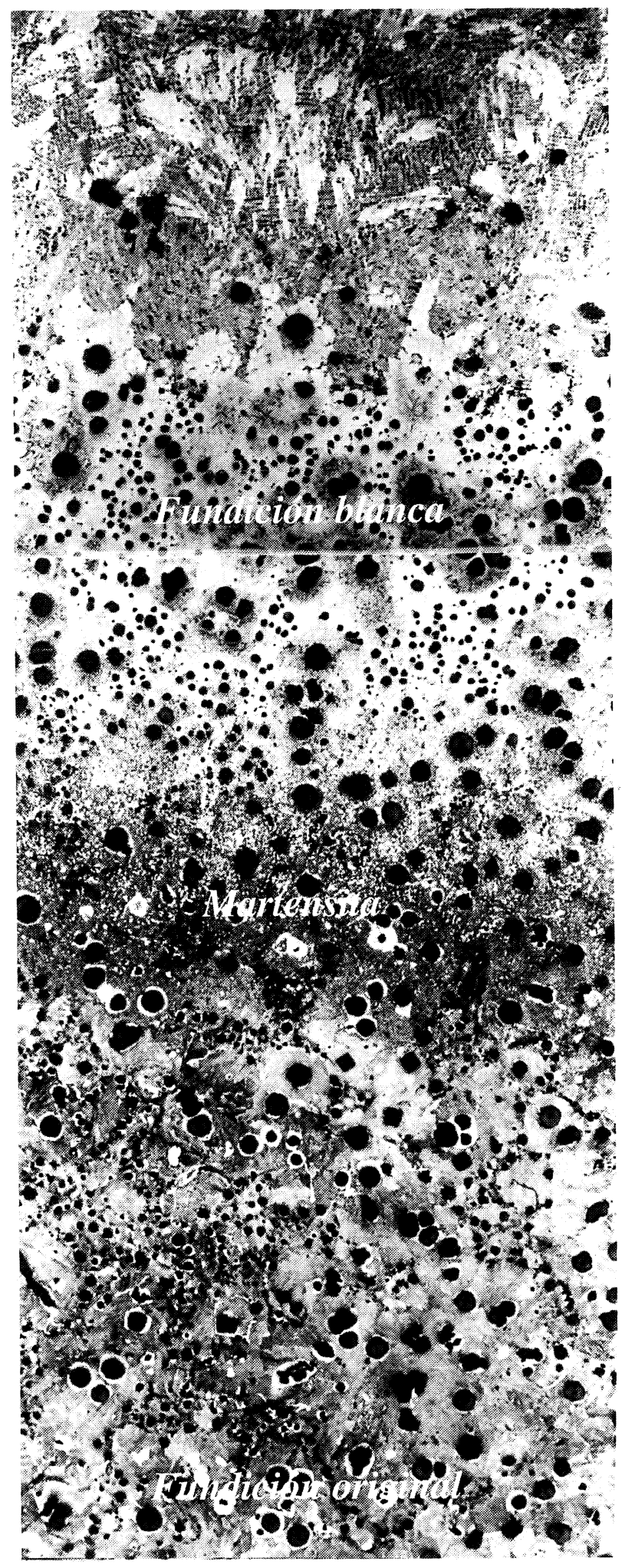

FIG. 4.- Alteración microestructural en la fundición nodular. $\times 50$.

FIG. 4.- Microstructural modification of the nodular gray iron. $\times 50$.

\subsection{Perfil de durezas}

Se ha visto que, a bajas velocidades, el espesor transformado involucra la aparición de dos regiones 
diferenciadas: una zona fundida (fundición blanca) y una zona templada (martensita). En ambos casos, tanto en la región en la que tiene lugar la transformación martensítica como en la que se logra la fusión, se consiguen notables aumentos de la dureza.

Las curvas de dureza muestran dos tramos claramente diferenciados, que corresponden a la estructura ledeburítica y martensítica, con durezas en torno a $1.000 \mathrm{HV}$ y $800 \mathrm{HV}$, respectivamente (Fig. 5). Mientras la dureza de las regiones martensíticas se ha mostrado independiente de la velocidad de pasada, la fundición blanca, obtenida con la velocidad más alta, era más dura que la generada con la velocidad más lenta, lo que se justifica en virtud de la microestructura ledeburítica más fina de aquella y su teóricamente menor contenido de austenita retenida.

De cualquier manera, el endurecimiento inducido por el tratamiento superficial con láser es siempre muy notable, dado que, como también se observa en la misma figura, la dureza de la fundición original es de $200 \mathrm{HV}$.

Como comentario final de todos los resultados obtenidos, podemos citar que la variación tanto del tipo de fundición como del diámetro del haz láser y de la velocidad de pasada afectan principalmente a la extensión de las regiones ledeburítica y martensítica obtenidas en cada caso, pero apenas influyen en la dureza final de las mismas.

\section{CONCLUSIONES}

1. El uso del modelo de flujo unidireccional ha mostrado ser una herramienta muy práctica a la

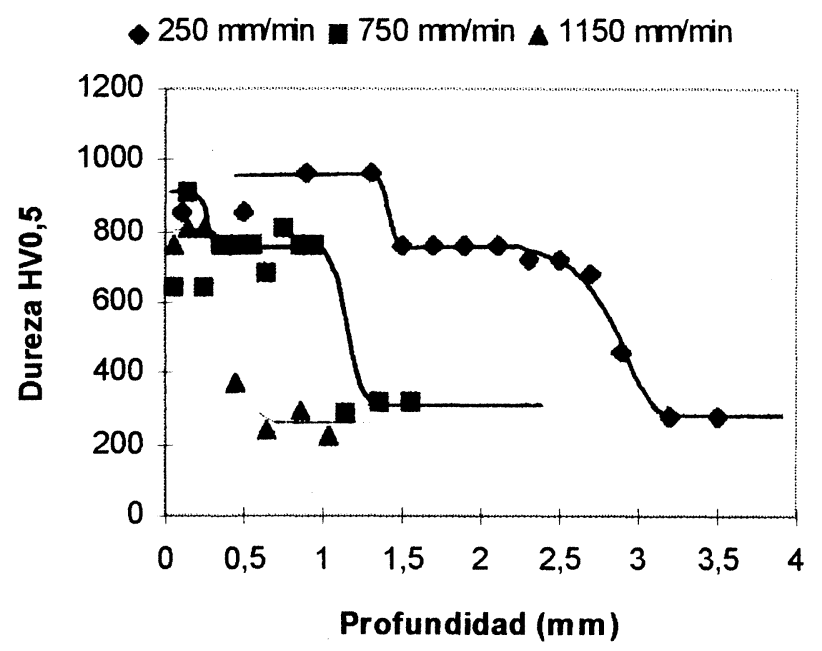

Fig. 5.- Evolución de la dureza en la fundición nodular aleada (NA) tratada con un haz de $14 \mathrm{~mm}$.

FIG. 5.-Hardness profile of a nodular alloyed iron (NA). Laser beam diameter: $14 \mathrm{~mm}$. hora de evitar probar arbitrariamente las condiciones en las que debe trabajar el láser; por tanto, se ahorra tiempo y recursos, puesto que el cálculo anticipado de los posibles espesores transformados condiciona un margen de las variables fundamentales del tratamiento, tales como la velocidad de pasada y el diámetro del haz láser, con los que necesariamente se producirán los cambios microestructurales deseados.

2. Las transformaciones estructurales que se generan al tratar superficialmente con láser los tres tipos de fundiciones son muy similares: cuando hay fusión aparece una microestructura ledeburítica, a su alrededor una interfase en la que está presente austenita retenida $\mathrm{y}$, a continuación, una zona martensítica y, finalmente, el material no alterado por el tratamiento. Cuando no hay fusión, la secuencia de microestructuras empieza en la zona martensítica.

3. Los valores de microdureza medidos en la región fundida son similares, aunque se observan pequeñas variaciones en función de las velocidades empleadas. En cambio, la dureza de la región martensítica es más independiente de las velocidades de movimiento del haz respecto a la pieza. Por otro lado, los valores de dureza de las regiones afectadas por el láser son muy altos e independientes del tipo de fundición.

\section{Agradecimiento}

Los autores agradecen la financiación recibida de la Fundación para el Fomento de la Investigación Científica y la Tecnología del Principado de Asturias (FICYT) y del Dpto. de Construcción e Ingeniería de Fabricación de la ETSI Industriales de Gijón (Universidad de Oviedo).

\section{REFERENCIAS}

(1) Макото, I. et al. Mater. Manuf. Proc . 12 (1), 1997: 37-46.

(2) Putatunda, S.K. et al. Mater. Manuf. Proc. 12 (1), 1997: 137-151.

(3) Materials Engineering: Materials Selector 1986. Penton Publ. (EE.UU). Dic. 1985: 30-31.

(4) ASTM G77-91. Vol 0302. ASTM. Filadelfia (EE.UU.), 1992: 310-321.

(5) Steen, W.M. Laser material processing. Springer-Verlag London Ltd. 1991: 150-151. 